\title{
Polyhedra with virtually polycyclic fundamental groups have finite depth
}

\author{
by
}

\author{
Danuta Kołodziejczyk (Warszawa)
}

\begin{abstract}
The notions of capacity and depth of compacta were introduced by K. Borsuk in the seventies together with some open questions. In a previous paper, in connection with one of them, we proved that there exist polyhedra with polycyclic fundamental groups and infinite capacity, i.e. dominating infinitely many different homotopy types (or equivalently, shapes). In this paper we show that every polyhedron with virtually polycyclic fundamental group has finite depth, i.e., there is a bound on the lengths of all descending sequences of different homotopy types (or shapes) dominated by this polyhedron. As a corollary, we deduce that for two ANR's with virtually polycyclic fundamental groups the so-called index of h-proximity, introduced by K. Borsuk in his monograph on retract theory, is finite. We also obtain an answer to some question of K. Borsuk concerning homotopy (or shape) decompositions of polyhedra into simple constituents.
\end{abstract}

1. Introduction. In 1979, at the International Topological Conference in Moscow, K. Borsuk introduced the capacity and depth in the shape category of compacta, together with some relevant questions (see [B1]). (The basic notions and results of shape theory can be found in [B4], [DS], [MS].)

Recall that a domination in a given category $\mathcal{C}$ is a morphism $f: X \rightarrow Y$, $X, Y \in \mathrm{Ob} \mathcal{C}$, for which there exists a morphism $g: Y \rightarrow X$ of $\mathcal{C}$ such that $f g=\mathrm{id}_{Y}$. Then we say that $Y$ is dominated by $X$, and we write $Y \leq X$ or $X \geq Y$; moreover, $X<Y$ will denote that $X \leq Y$ holds but $Y \leq X$ fails.

In the following, $\mathcal{C}$ is the homotopy category of $\mathrm{CW}$-complexes and homotopy classes of cellular maps between them or the shape category of compacta (pointed or unpointed).

2000 Mathematics Subject Classification: 55P15, 55P55, 55P10.

Key words and phrases: polyhedron, ANR, CW-complex, compactum, homotopy domination, homotopy type, shape domination, shape, depth, index of h-proximity, simple constituent.

Research partially supported by the Ministry of Sciences and Higher Education grant \# 1 P03A 00530. 
Following K. Borsuk (cf. [B1]), define the capacity $C(A)$ of an $A \in \mathrm{Ob} \mathcal{C}$ as the cardinality of the class of isomorphism classes of all the $X \in \mathrm{Ob} \mathcal{C}$ such that $X \leq A$.

A system $X_{1}<\cdots<X_{k} \leq A$, where $X_{i} \in \mathrm{Ob} \mathcal{C}$ for $i=1, \ldots, k$, is called a chain of length $k$ for $A \in \mathrm{ObC}$. The depth $D(A)$ of $A$ is the least upper bound of the lengths of all chains for $A$. If this upper bound is infinite, we write $D(A)=\aleph_{0}$ (cf. [B1]).

In other words, $A \in \mathrm{ObC}$ has finite depth if and only if there exists an integer $k$ such that each sequence $\cdots \leq X_{i} \leq \cdots \leq X_{1} \leq A$ contains at most $k$ different objects (up to isomorphism).

It is clear that $D(A) \leq C(A)$ for each $A \in \mathrm{ObC}$.

In the following, we will assume (without loss of generality) that every polyhedron, ANR, and CW-complex considered is connected.

Note that for a polyhedron, the capacity and depth in the shape category of compacta are the same as in the homotopy category of CW-complexes and homotopy classes of cellular maps between them. Indeed, there is a 1-1 functorial correspondence between the shapes of compacta shape dominated by a given polyhedron and the homotopy types of CW-complexes homotopy dominated by it (see [DS, Theorem 2.2.6], [HaHe1] or [HaHe2], [EG]). Similarly, the capacity (and depth) of a given polyhedron is the same in both pointed and unpointed cases. This follows from [D, Theorem 5.1].

In 1968 at the Topological Conference in Herceg-Novi K. Borsuk stated the question: Is the capacity of each polyhedron finite? (see, for example, [B1, Question 4]).

By the classical results of shape theory, this was known to be the case for 1-dimensional polyhedra (see [Tr] and [B4, Theorem 7.1, p. 221]). In general the answer to Borsuk's question is negative: there exist polyhedra (even of dimension 2) homotopy dominating infinitely many different homotopy types, and equivalently, shapes ([K6], see also [K7]). Furthermore, there exist polyhedra with polycyclic (and even nilpotent) fundamental groups dominating infinitely many different homotopy types or shapes ([K1]).

On the other hand, all the simply-connected polyhedra, polyhedra with finite fundamental groups and nilpotent polyhedra have finite capacity, hence also finite depth ([K5], [K4], [K3]).

The main result of this paper is that although there exist polyhedra with polycyclic fundamental groups with infinite capacity, every polyhedron with virtually polycyclic fundamental group has finite depth.

This result, together with the previous ones from [K1], will enable us to give a positive answer to a question of Borsuk from [B2]: Does there exist a polyhedron with infinitely many simple constituents? Recall that $X$ and $Y$ are said to be constituents of the wedge (one-point union) $X \vee Y$ (cf. [B2]). 
$X$ is called simple if each of its constituents is either trivial or coincides with $X$ in $\mathcal{C}$.

In his monograph [B3] on retract theory, K. Borsuk introduced the notions of h-neighbors and the index of h-proximity of two ANR's.

The homotopy type of $X$ is a left h-neighbor of the homotopy type of $Y$ if $X<Y$ and for every $Z$ such that $X \leq Z \leq Y, X \simeq Z$ or $Y \simeq Z$ (see [B3, p. 349]). In the same manner we define a right h-neighbor of the homotopy type of $Y$.

We say that the index of h-proximity of two ANR's $P$ and $Q, h(P, Q)$, is equal to $n$ if there exists a sequence $P=X_{0}, X_{1}, \ldots, X_{n}=Q$ such that $X_{i}$ and $X_{i+1}$ are h-neighbors. If no such finite sequence exists then we say that $h(P, Q)$ is infinite (see [B3, Ch. IX, 12]).

Concerning these notions, Borsuk asked (1967, [B3, Ch. IX, Problem (12.8)]): Do there exist two ANR's, $P$ and $Q$, for which $h(P, Q)$ is infinite?

This question remains open. In the second part of this paper we will show, as another corollary to the main result, that the index of h-proximity of two ANR's with virtually polycyclic fundamental groups is finite.

\section{Algebraic preliminaries}

Definition. Recall that a group $G$ is polycyclic if it has a finite series of subgroups $G=G_{0} \triangleright G_{1} \triangleright \cdots \triangleright G_{l}=1$ for which each factor $G_{i-1} / G_{i}$ (where $i=1, \ldots, l$ ) is finite cyclic or infinite cyclic (see, for example, [S, p. 2]).

We say that $G$ is a poly-ZZ-group if it has such a series with factors $\mathbb{Z}$.

Definition. A group $G$ is called virtually polycyclic if there exists a polycyclic group $H \triangleleft G$ such that $G / H$ is finite.

Definition. The number $h(G)$ of infinite cyclic factors in a series $G=$ $G_{0} \triangleright G_{1} \triangleright \cdots \triangleright G_{l}=1$ with cyclic or finite factors is an invariant of a virtually polycyclic group $G$ (independent of the series), known as the Hirsch number of $G$ (see [S, p. 16]).

Definition. Suppose that $\mathcal{P}$ is a property of groups. A group $G$ is called poly- $\mathcal{P}$ if it has a finite series of subgroups $G=G_{0} \triangleright G_{1} \triangleright \cdots \triangleright G_{l}=1$ for which each factor $G_{i-1} / G_{i}$ (where $i=1, \ldots, l$ ) has the property $\mathcal{P}$ ([S, p. 2]).

Definition. Let $R$ be a ring. A right $R$-module $M$ is said to be noetherian if every $R$-submodule of $M$ is finitely generated, equivalently, $M$ satisfies the ascending chain condition, i.e., every ascending sequence $M_{1} \subsetneq$ $M_{2} \subsetneq \cdots$ of submodules of $M$ is finite (cf. [Pa, p. 419], or [La]).

We call a ring $R$ noetherian if it is a noetherian module as a module over itself.

Definition. Let $R$ be a ring and $M$ be a right $R$-module. The Krull dimension of $M$, denoted by $\operatorname{Kdim} M$, is defined by transfinite recursion as 
follows: if $M=0$, then $\operatorname{Kdim} M=-1$; if $\alpha$ is an ordinal and $\operatorname{Kdim} M \nless \alpha$, then $\operatorname{Kdim} M=\alpha$ provided there is no infinite descending chain $M=M_{0} \supset$ $M_{1} \supset \cdots$ of submodules $M_{i}$ such that $\operatorname{Kdim} M_{i-1} / M_{i} \nless \alpha$ for $i=1,2, \ldots$. If there is no ordinal $\alpha$ such that $\operatorname{Kdim} M=\alpha$, we say that $M$ has no Krull dimension (see [GR, p. 5] or $[\mathrm{Br}]$ ).

DEFINITION. If a module contains no infinite direct sum of nonzero submodules, then there is a fixed bound on the number of summands in finite sums it can contain; and the module is said to have finite uniform dimension (see [GR, p. 7]).

DeFinition. Recall that in the category of groups or modules with homomorphisms, a homomorphism $f: A \rightarrow B$ is said to be an $r$-homomorphism if there exists a homomorphism $g: B \rightarrow A$ such that $f g=\mathrm{id}_{B}$ (see, for example, [B3, Ch. 2]).

\section{Polyhedra with virtually polycyclic fundamental groups have} finite depth. To prove that every polyhedron with virtually polycyclic fundamental group has finite depth, we will use properties of finitely generated noetherian modules over the integral group rings $\mathbb{Z} G$, where $G$ is virtually polycyclic, and the concept of Krull dimension of modules. We begin with some algebraic lemmas.

Lemma 1 (cf. Lemma 1 from [K1]). Let $G$ be a virtually polycyclic group. There exists an integer $k_{G}$ such that any sequence $G=G_{0} \supseteq G_{1} \supseteq \cdots \supseteq G_{l}$ of subgroups of $G$ with $r$-homomorphisms $r_{i}: G_{i-1} \rightarrow G_{i}$ for $i=1, \ldots, l$ contains no more than $k_{G}$ distinct subgroups.

Proof. Note that every subgroup of a virtually polycyclic group is also virtually polycyclic (cf. [S, Ex. 3, p. 2]). If $h(G)$ is the Hirsch number of the virtually polycyclic group $G, H \subseteq G$ and $N \triangleleft G$ is such that $H \cong G / N$, then $h(G)=h(N)+h(H)$ (see, for example, [S, Ex. 8, p. 16]).

Let $N_{i}$ be the kernel of $r_{i}: G_{i-1} \rightarrow G_{i}$. Then $N_{i}$ is a normal subgroup of $G_{i-1}$ which satisfies $G_{i-1}=G_{i} N_{i}$ and $G_{i} \cap N_{i}=1$ for $i=1, \ldots, l$. It follows that $h\left(G_{i-1}\right)=h\left(N_{i}\right)+h\left(G_{i}\right)$ for $i=1, \ldots, l$. Therefore $h\left(G_{i-1}\right)=h\left(G_{i}\right)$, hence $h\left(N_{i}\right)=0$, for all $i$ except at most $h(G)$.

Assume, without loss of generality, that $h\left(N_{i}\right)=0$ for all $i \geq 2$. It follows that the $N_{i}$ are all finite. Indeed, if a virtually polycyclic group has Hirsch number 0 , then it is finite (for example by [S, p. 16], $h(H)=h(K)$ if and only if $|K: H|<\infty)$.

There are only at most $l_{G}$, where $l_{G}$ is an integer depending only on $G$, possible different finite subgroups $N_{i}$ in $G$. This follows from the fact that for each integer $l \geq 2, N_{l} \cdot N_{l-1} \cdot \ldots \cdot N_{2}$ is a finite subgroup of $G$ (apply [Hu, Ch. I, Theorem 5.3, p. 42]) of rank $\left|N_{l}\right| \cdot\left|N_{l-1}\right| \cdot \ldots \cdot\left|N_{2}\right|$ (by [Hu, Ch. I, Theorem 4.7, p. 39]). On the other hand, a given virtually polycyclic group has only 
finitely many finite subgroups up to isomorphism (hence the ranks of finite subgroups are bounded). This can be deduced, for example, from the fact that a group is virtually polycyclic if and only if it is (poly- $\mathbb{Z}$ )-by-finite (see [S, Proposition 2, p. 2]).

Thus there exists an integer $k_{G}$ such that $G_{i}=G_{i-1}$ for all $i$ except at most $k_{G}$. This finishes the proof.

The next lemma is an analog of Lemma 1 for finitely generated modules over the integral group ring $\mathbb{Z} G$ of a virtually polycyclic group $G$.

Lemma 2. Let $M$ be a finitely generated module over the integral group ring $\mathbb{Z} G$, where $G$ is a virtually polycyclic group. Then there exists an integer $k_{M}$ such that any sequence $M=M_{0} \supseteq M_{1} \supseteq \cdots \supseteq M_{l}$ of $\mathbb{Z} G$-submodules of $M$ with r-homomorphisms $R_{i}: M_{i-1} \rightarrow M_{i}$ for $i=1, \ldots, l$ contains no more than $k_{M}$ distinct modules.

Proof. If $G$ is a virtually polycyclic group then the group $\operatorname{ring} \mathbb{Z} G$ is right noetherian, by the result of P. Hall (see [R, (15.3.3), p. 446]). Therefore, every finitely generated module over $\mathbb{Z} G$ is noetherian (see [La, Ch. VI] and Introduction). Thus $M$ is noetherian.

Consider a sequence $M=M_{0} \supseteq M_{1} \supseteq \cdots \supseteq M_{l}$ of $\mathbb{Z} G$-modules with r-homomorphisms $R_{i}: M_{i-1} \rightarrow M_{i}$ for $i=1, \ldots, l$.

Let $N_{i}=\operatorname{ker} R_{i}$. We have $M_{i-1} \cong M_{i} \oplus N_{i}$ for $i=1, \ldots, l$. Thus

$$
M_{0} \cong N_{1} \oplus \cdots \oplus N_{l} \oplus M_{l} .
$$

It was proved by Gabriel ([Ga], see also [GR, Proposition 1.3, p. 7]) that every noetherian module has Krull dimension. It was also shown by Krause $[\mathrm{Kr}]$ and Michler [Mi] that a module with Krull dimension has finite uniform dimension (see [GR, Proposition 1.4, p. 7]). Therefore, there is a fixed bound on the number of possible direct summands of $M=M_{0}$. It follows that there exists an integer $k_{M}$ such that $N_{i}=0$ for all except no more than $k_{M}$ integers $i$. This means that $M_{i-1}=M_{i}$ for such $i$, and the proof is finished.

In this paper $\tilde{X}$ denotes, as usual, the universal covering space of $X$.

Let $f: X \rightarrow Y$ be a cellular map of CW-complexes such that $f(x)=y$ for some vertices $x \in X, y \in Y$. Choose $\widetilde{x} \in p^{-1}(x), \widetilde{y} \in p^{-1}(y)$ (where $p$ denotes the covering projections). There exists a unique map $\widetilde{f}: \widetilde{X} \rightarrow \widetilde{Y}$ such that $p \widetilde{f}=f p$ and $\widetilde{f}(\widetilde{x})=\widetilde{y}$. The map $\widetilde{f}$ induces homomorphisms $\widetilde{f}_{r}: H_{r}(\widetilde{X}) \rightarrow H_{r}(\widetilde{Y})$ for all $r \geq 1$. We then say that $\widetilde{f}_{r}$ is induced by $f$ (see [Hl, p. 107]).

Applying the above algebraic results we obtain (cf. [K2, Theorem 1])

THEOREM 1. Let $P$ be a polyhedron with virtually polycyclic fundamental group. There exists an integer $k_{P}$ such that each sequence

$$
P \geq X_{1} \geq \cdots \geq X_{l}
$$


of spaces contains at most $k_{P}$ homotopy dominations which are not homotopy equivalences.

Proof. Recall that by the classical result of J. H. C. Whitehead, each space homotopy dominated by a finite CW-complex has the homotopy type of a CW-complex, not necessarily finite (see also [Wa]). Thus assume $X_{i}$ for $i=1, \ldots, l$ to be $\mathrm{CW}$-complexes.

We will prove that if $P$ is a polyhedron with virtually polycyclic fundamental group, then there exists an integer $k_{P}$ such that for each sequence $P=X_{0} \geq X_{1} \geq \cdots \geq X_{l}$ of CW-complexes, with dominations $d_{i}: X_{i-1} \rightarrow X_{i}$ and inverse maps $u_{i}: X_{i} \rightarrow X_{i-1}$ (i.e. $d_{i} u_{i} \simeq \operatorname{id}_{X_{i}}$ ) for $i=1, \ldots, l$, all except at most $k_{P}$ dominations (and their inverses) are homotopy equivalences.

Let $\pi_{1}(P) \cong G$. By Lemma 1 , there exists an integer $k_{G}$ such that for each sequence $P \geq X_{1} \geq \cdots \geq X_{l}$ all except at most $k_{G}$ dominations $d_{i}: X_{i-1} \rightarrow X_{i}$ (and their inverses $u_{i}: X_{i} \rightarrow X_{i-1}$ ) induce isomorphisms of $\pi_{1}\left(X_{i}\right)$.

Let $\widetilde{d}_{i}: \widetilde{X}_{i-1} \rightarrow \widetilde{X}_{i}$ and $\widetilde{u}_{i}: \widetilde{X}_{i} \rightarrow \widetilde{X}_{i-1}$ be the liftings of $d_{i}$ and $u_{i}$ to the universal covers. Then $\widetilde{d}_{i}$ is a domination.

For each $2 \leq r \leq \operatorname{dim} P$, we will apply Lemma 2 to the $\mathbb{Z} G$-module $M=H_{r}(\widetilde{P})$.

From [Wa, Theorems $\mathrm{A}$ and $\mathrm{B}]$, if $\mathbb{Z} H$ is a noetherian ring, then for every CW-complex $X$ with $\pi_{1}(X) \cong H$ which is dominated by a finite CW-complex, the $\mathbb{Z} H$-modules $H_{r}(\tilde{X})$ for $r \geq 2$ are all finitely generated.

Since $\mathbb{Z} G$ is noetherian (cf. the proof of Lemma 2), $M$ is a finitely generated $\mathbb{Z} G$-module. Consider the sequence $M=M_{0} \supseteq M_{1} \supseteq \cdots \supseteq M_{l}$ of $\mathbb{Z} G$-modules in which

$$
M_{i}=\widetilde{u}_{1 *} \widetilde{u}_{2 *} \cdots \widetilde{u}_{i *}\left(H_{r}\left(\widetilde{X}_{i}\right)\right) \quad \text { for } i=1, \ldots, l,
$$

where $\widetilde{u}_{i *}: H_{r}\left(\widetilde{X}_{i}\right) \rightarrow H_{r}\left(\widetilde{X}_{i-1}\right)$ is induced by $\widetilde{u}_{i}: \widetilde{X}_{i} \rightarrow \widetilde{X}_{i-1}$ (with obvious r-homomorphisms between them).

By Lemma 2, there exists an integer $k_{r}$ (depending only on $P$ and $r$ ) such that $M_{i}=M_{i-1}$ for all except at most $k_{r}$ integers $i$. It follows that in each sequence

$$
H_{r}(\widetilde{P})=H_{r}\left(\widetilde{X}_{0}\right) \rightarrow H_{r}\left(\widetilde{X}_{1}\right) \rightarrow \cdots \rightarrow H_{r}\left(\widetilde{X}_{l}\right)
$$

with r-homomorphisms $\widetilde{d}_{i *}: H_{r}\left(\widetilde{X}_{i-1}\right) \rightarrow H_{r}\left(\widetilde{X}_{i}\right)$ induced by $\widetilde{d}_{i}: \widetilde{X}_{i-1} \rightarrow \widetilde{X}_{i}$, the homomorphisms are all isomorphisms except at most $k_{r}$ (and the same is true for $\widetilde{u}_{i *}: H_{r}\left(\widetilde{X}_{i}\right) \rightarrow H_{r}\left(\widetilde{X}_{i-1}\right)$ induced by $\left.\widetilde{u}_{i}: \widetilde{X}_{i} \rightarrow \widetilde{X}_{i-1}\right)$.

Hence, for a given polyhedron $P$ with virtually polycyclic fundamental group, there exists an integer $k_{P}$ such that, for each sequence

$$
P=X_{0} \geq X_{1} \geq \cdots \geq X_{l}
$$


of CW-complexes with dominations $d_{i}: X_{i-1} \rightarrow X_{i}, d_{i}$ induce isomorphisms $\pi_{1}\left(X_{i-1}\right) \rightarrow \pi_{1}\left(X_{i}\right)$ and $H_{r}\left(\widetilde{X}_{i-1}\right) \rightarrow H_{r}\left(\widetilde{X}_{i}\right)$ (for all $r \geq 2$ ) for all $i=$ $1, \ldots, l$ except at most $k_{P}$.

Recall that, by the Whitehead theorem, if $X$ and $Y$ are CW-complexes, then a map $f: X \rightarrow Y$ which induces an isomorphism $\pi_{1}(X) \rightarrow \pi_{1}(Y)$ and isomorphisms $H_{r}(\widetilde{X}) \rightarrow H_{r}(\tilde{Y})$ for all $r \geq 2$ is a homotopy equivalence (see, for example, [Hl, Theorem 3.1, p. 107]).

Thus, for a polyhedron $P$ with virtually polycyclic fundamental group, there exists an integer $k_{P}$ such that each sequence $P \geq X_{1} \geq \cdots \geq X_{l}$ of $\mathrm{CW}$-complexes contains at most $k_{P}$ homotopy dominations which are not homotopy equivalences, which is the desired conclusion.

THEOREM 2. Every polyhedron with virtually polycyclic fundamental group has finite depth.

Proof. This follows from Theorem 1.

Corollary 1. Let $P$ be a polyhedron with virtually polycyclic fundamental group. There exists an integer $k_{P}$ such that each sequence $P \geq X_{1} \geq$ $X_{2} \geq \cdots$ contains at most $k_{P}$ homotopy dominations which are not homotopy equivalences.

COROLlary 2. Let $P$ be a polyhedron with virtually polycyclic fundamental group. There exists an integer $k_{P}$ such that each sequence $X_{1} \leq$ $X_{2} \leq \cdots$ with $X_{i} \leq P$ for each $i$ contains at most $k_{P}$ homotopy dominations which are not homotopy equivalences.

4. The index of h-proximity of two ANR's with virtually polycyclic fundamental groups is finite. In [B3] K. Borsuk introduced the notions of h-neighbor, left h-neighbor, right h-neighbor, h-minorant, h-majorant, and the index of h-proximity of two ANR's. Some results concerning these notions can be found in $[\mathrm{M}]$. In $[\mathrm{K} 7]$ we gave an example of a polyhedron with infinitely many left neighbors (in the shape or homotopy category), which answers Borsuk's question from [B4, p. 349].

The following problem of K. Borsuk (1967) is still open:

Problem [B3, Ch. IX, Problem (12.8)]. Do there exist two ANR's, P and $Q$, for which the index of h-proximity $h(P, Q)$ is infinite?

As a corollary to Theorem 1, we find that if the fundamental groups of two ANR's are virtually polycyclic, then their index of h-proximity is finite.

TheOREM 3. Assume that $P, Q \in \mathrm{ANR}$ have $\pi_{1}(P)$ and $\pi_{1}(Q)$ virtually polycyclic. Then $h(P, Q)$ is finite.

Proof. Set $W=P \times Q \in$ ANR. Obviously $P \leq W$ and $Q \leq W$. 
Since a group is poly-(cyclic or finite) if and only if it is virtually polycyclic [S, Proposition 2, p. 2], $\pi_{1}(W) \cong \pi_{1}(P) \times \pi_{1}(Q)$ is virtually polycyclic.

Note that if $X, Y \leq W$, where $W$ is a polyhedron and $\pi_{1}(W)$ is virtually polycyclic, then $X \geq Y$ and $Y \geq X$ imply that $X \simeq Y$ (by Corollary 1).

To prove the theorem, it suffices to show that there exist finite sequences $P=X_{0} \leq X_{1} \leq \cdots \leq X_{s}=W$ and $Q=Y_{0} \leq Y_{1} \leq \cdots \leq Y_{t}=W$ such that $X_{i} \leq Z \leq X_{i+1}$ implies $Z \simeq X_{i}$ or $Z \simeq X_{i+1}$, where $i=0, \ldots, s-1$, and $Y_{i} \leq Z \leq Y_{i+1}$ implies $Z \simeq Y_{i}$ or $Z \simeq Y_{i+1}$, where $i=0, \ldots, t-1$, respectively.

From Theorem 1 we infer that there exists an integer $k_{P}$ such that each sequence $P \leq X_{1} \leq \cdots \leq X_{s} \leq W$ contains at most $k_{P}$ different homotopy types. We also have an analog of this assertion for $Q$ in place of $P$. This completes the proof.

5. There exist polyhedra with infinitely many simple constituents. In $1970 \mathrm{~K}$. Borsuk asked:

Problem ([B2, Problem 7]). Is it true that for every $P \in \mathrm{ANR}$, the shape $\operatorname{Sh}(P, p)$ has only a finite number of simple constituents?

Applying the main result of this paper, we can answer this question:

THEOREM 4. There exists a polyhedron (with polycyclic fundamental group) with infinitely many simple constituents.

The proof is based on the following corollary to Theorem 2 :

Lemma 3. Let $P$ be a polyhedron with virtually polycyclic fundamental group. Then there exists an integer $l_{P}$ such that $P$ cannot be decomposed into more than $l_{P}$ constituents.

Proof. Let $P \simeq Y_{1} \vee \cdots \vee Y_{l}$, where each $Y_{i}$ has nontrivial homotopy type. Set $X_{i}=Y_{1} \vee \cdots \vee Y_{i}$ for $i=1, \ldots, l$. Then $X_{1} \leq \cdots \leq X_{l}$. By Theorem 1 , there exists an integer $k_{P}$ such that all except at most $k_{P}$ dominations in this sequence are homotopy equivalences. On the other hand, $X_{i} \simeq X_{i+1}$ would imply that there exists a domination of $X_{i}$ over itself which is not a homotopy equivalence. But this is impossible (see Corollary 1). Therefore $l_{P}=k_{P}+1$, which ends the proof.

Proof of Theorem 4. In [K1] we proved that there exist polyhedra $P$ with polycyclic fundamental groups such that $P \simeq K_{i} \vee S^{3}$ for infinitely many polyhedra $K_{i}$ of different homotopy types.

By Lemma 3 , there exists an integer $l_{P}$ such that no $K_{i}$ can be decomposed into more than $l_{P}$ simple constituents. Since the $K_{i}$, for $i=1,2, \ldots$, represent infinitely many different homotopy types, it is easily seen that $P$ has infinitely many simple constituents with different homotopy types, and the proof is finished. 


\section{Final remarks. Let us state the following:}

Problem 1. Does there exist a polyhedron with infinite depth?

We suspect that the answer to this question is positive and that the example is possible even with soluble fundamental group. One may ask about other classes of polyhedra with finite depth. It is worth pointing out that the following questions of a similar nature, included in known lists of open problems, also remain unsolved:

Problem 2 (Y. Rong, [RWZ]). Let $M$ and $M_{i}$ be closed, orientable, aspherical 3-manifolds. Does there exist an integer $N_{M}$ such that any sequence

$$
\pi_{1}(M) \rightarrow \pi_{1}\left(M_{1}\right) \rightarrow \pi_{1}\left(M_{2}\right) \rightarrow \cdots \rightarrow \pi_{1}\left(M_{n}\right)
$$

of epimorphisms with $n \geq N_{M}$ contains an isomorphism?

Problem 3 (Y. Rong, [Kb, Problem 3.100]). Let $M$ and $M_{i}$ be closed, orientable 3-manifolds. Does there exist an integer $N_{M}$ such that any sequence

$$
M \rightarrow M_{1} \rightarrow M_{2} \rightarrow \cdots \rightarrow M_{n}
$$

of degree 1 maps with $n \geq N_{M}$ contains a homotopy equivalence?

Problem 4 (J. Simon, [Kb, Problem 1.12]). For a knot $K$ in $S^{3}$, let $G_{K}=\pi_{1}\left(S^{3} \backslash K\right)$. Given $K$, does there exist an integer $N_{K}$ such that any sequence

$$
G_{K} \rightarrow G_{K_{1}} \rightarrow G_{K_{2}} \rightarrow \cdots \rightarrow G_{K_{n}}
$$

of epimorphisms of knot groups with $n \geq N_{K}$ contains an isomorphism?

\section{References}

[B1] K. Borsuk, Some problems in the theory of shape of compacta, Russian Math. Surveys 34 (1979), no. 6, 24-26.

[B2] - , Some remarks concerning the shape of pointed compacta, Fund. Math. 67 (1970), 221-240.

[B3] -, Theory of Retracts, Monograf. Mat. 44, Polish Sci. Publ., Warszawa, 1967.

[B4] - Theory of Shape, Monograf. Mat. 59, Polish Sci. Publ., Warszawa, 1975.

[Br] C. J. B. Brookes, Finitely presented groups and the finite generation of exterior powers, in: London Math. Soc. Lecture Note Ser. 204, Cambridge Univ. Press, 1993, 16-28.

[D] J. Dydak, Pointed and unpointed shape and pro-homotopy, Fund. Math. 107 (1980), 58-69.

[DS] J. Dydak and J. Segal, Shape Theory: An Introduction, Lecture Notes in Math. 688, Springer, Berlin, 1978.

[EG] D. A. Edwards and R. Geoghegan, Shapes of complexes, ends of manifolds, homotopy limits and the Wall obstruction, Ann. of Math. (2) 101 (1975), 521-535.

[Ga] P. Gabriel, Des catégories abéliennes, Bull. Soc. Math. France 90 (1960), 323-448.

[GR] R. Gordon and J. C. Robson, Krull dimension, Mem. Amer. Math. Soc. 133 (1973).

[HaHe1] H. M. Hastings and A. Heller, Homotopy idempotents on finite-dimensional complexes split, Proc. Amer. Math. Soc. 85 (1982), 619-622. 
[HaHe2] H. M. Hastings and A. Heller, Splitting homotopy idempotents, in: Shape Theory and Geometric Topology (Dubrovnik, 1981), Lecture Notes in Math. 870, Springer, Berlin, 1981, 25-36.

[Hl] P. Hilton, An Introduction to Homotopy Theory, Cambridge Univ. Press, 1961.

[Hu] T. W. Hungerford, Algebra, Holt, Rinehart and Winston, New York, 1974.

[Kb] R. Kirby, Problems in low dimensional topology, in: Geometric Topology, W. H. Kazez (ed.), AMS/IP Vol. 2, Amer. Math. Soc. and Internat. Press, 1997, 35-473.

[K1] D. Kołodziejczyk, Homotopy dominations within polyhedra, Fund. Math. 178 (2003), 189-202.

[K2] - Homotopy dominations by polyhedra with polycyclic-by-finite fundamental groups, Topology Appl. 153 (2005), 294-302.

[K3] - Polyhedra dominating finitely many different homotopy types, ibid. 146-147 (2005), 583-592.

[K4] - Polyhedra with finite fundamental group dominate finitely many different homotopy types, Fund. Math. 180 (2003), 1-9.

[K5] - Simply-connected polyhedra dominate only finitely many different shapes, Topology Appl. 112 (2001), 289-295.

[K6] - There exists a polyhedron dominating infinitely many polyhedra of different homotopy types, Fund. Math. 151 (1996), 39-46.

[K7] - There exists a polyhedron with infinitely many left neighbors, Proc. Amer. Math. Soc. 129 (2001), 303-309.

[Kr] G. Krause, On the Krull-dimension of left noetherian left Matlis-rings, Math. Z. 118 (1970), 207-214.

[La] S. Lang, Algebra, Addison-Wesley, Reading, MA, 1965.

[MS] S. Mardešić and J. Segal, Shape Theory. The Inverse System Approach, NorthHolland Math. Library 26, North-Holland, Amsterdam, 1982.

[Mi] G. Michler, Primringe mit Krull-dimension eins, J. Reine Angew. Math. 239/240 (1970), 366-381.

[M] M. Moszyńska, On the homotopy classification of spaces, Fund. Math. 66 (1969), 65-83.

[Pa] D. Passman, The Algebraic Structure of Group Rings, Wiley, New York, 1977.

[R] D. J. S. Robinson, A Course in the Theory of Groups, Springer, 1982.

[RWZ] A. W. Reid, S. Ch. Wang and Q. Zhou, Generalized Hopfian property, a minimal Haken manifold, and epimorphisms between 3-manifold groups, Acta Math. Sinica 18 (2002), 157-172.

[S] D. Segal, Polycyclic Groups, Cambridge Tracts in Math. 82, Cambridge Univ. Press, 1983.

[Tr] S. Trybulec, On shapes of movable curves, Bull. Acad. Polon. Sci. 21 (1973), 727-733.

[Wa] C. T. C. Wall, Finiteness conditions for $C W$-complexes, Ann. of Math. 81 (1965), 56-69.

Faculty of Mathematics and Information Science

Warsaw University of Technology

Pl. Politechniki 1, 00-661 Warszawa, Poland

E-mail: dkolodz@mimuw.edu.pl

Received 31 January 2006;

in revised form 23 October 2007 